\title{
Generalized Fermi acceleration
}

\author{
Martin Lemoine* \\ Institut d'Astrophysique de Paris \\ CNRS - Sorbonne Université \\ 98 bis boulevard Arago, F-75014 Paris, France \\ E-mail: lemoinediap.fr
}

This paper introduces a new formalism to describe the acceleration of particles in highly conducting astrophysical plasmas. This method tracks the evolution of particle momenta in a sequence of locally comoving reference frames, where the electric field exactly vanishes. It provides an efficient characterization of acceleration in sub- and ultra-relativistic settings, in Cartesian or non-Cartesian geometries, flat or non-flat spacetime. This paper provides three concrete, original applications: acceleration in a magnetized expanding wind from a compact object, acceleration in the vicinity of a black hole, and acceleration in a relativistic magnetized turbulence.

HEPRO VII

9 - 12 July, 2019

University of Barcelona (Barcelona, Spain)

${ }^{*}$ Speaker. 


\section{Introduction}

Non-thermal emission from astrophysical sources, routinely observed from radio frequencies to the very high-energy gamma-ray domain, generically results from radiative emission processes of particles that have been accelerated to high, possibly very high, energies.

In the realm of highly conducting astrophysical plasmas, particle acceleration proceeds in motional electric fields $\boldsymbol{E}$, which are related to their magnetic field counterpart $\boldsymbol{B}$ and to the plasma velocity $\boldsymbol{v}_{\mathrm{p}}$ by the ideal Ohm's law, $\boldsymbol{E}=-\boldsymbol{v}_{\mathrm{p}} \times \boldsymbol{B} / \boldsymbol{c}$. Accordingly, such electric fields vanish in the plasma rest frame (where $\boldsymbol{v}_{\mathrm{p}}=0$ ), hence particles cannot be accelerated there, but simply deflected by the magnetic field. Consequently, the particle cannot steadily gain energy, in any frame, if the velocity field is uniform throughout space. The guiding principle of Fermi-type acceleration scenarios relies on the observation that, if the velocity field is not uniform, one cannot define a global reference frame in which the electric field vanishes everywhere, hence actual energization becomes possible [1].

One commonly distinguishes various schemes of Fermi acceleration, e.g. stochastic (Fermi-II) acceleration in turbulence [2], diffusive shock (Fermi-I) acceleration [3], shear acceleration [4] etc., but, in truth, they only differ one from the other in how the velocity field is distributed throughout space. In order to describe the physics of acceleration, and more specifically, to evaluate the acceleration timescale $t_{\text {acc }}$ which is widely used in phenomenological applications, various methods have been proposed in the literature, with quite often a specific method applied to a given setting.

The present paper presents an original method to characterize Fermi-type acceleration, which has the advantage of being more versatile than other methods, in the sense that it can apply to any scenario, to sub- or ultra-relativistic flow velocities, and curved or flat geometries (Sec. 2). In some cases, it provides a simpler way to quantify how, and to what extent, the particle can gain energy. As applications, the following discusses three original cases, so far largely unexplored in the literature: stochastic unipolar induction in a magnetized wind from a compact object (Sec. 3.1), Fermi-type acceleration in black hole environments (Sec. 3.2) and stochastic acceleration in relativistic turbulence (Sec. 3.3). The present discussion follows that in [5], on which it is based.

\section{Formalism}

The idea behind the present method is to track the particle momentum in a sequence of comoving frames, defined along the particle trajectory, in which the electric field exactly vanishes. At any point $x$ of spacetime, one can indeed define an instantaneous frame of rest through a deboost by the four-velocity $\boldsymbol{u}(\mathrm{x})=\boldsymbol{E} \times \boldsymbol{B} /\left(B^{2}-E^{2}\right)$. Because the velocity field is not uniform, this change of reference frame is not global and is not achieved through a Lorentz transform, but rather through the definition of a local accelerated frame, written $\mathscr{R}_{\mathrm{u}}$ in the following. This can be done through the construction of an orthonormal tetrad, which is more conveniently characterized by the vierbeins $e^{\hat{a}}{ }_{\mu}$, which relate the components of four-vectors in the laboratory frame $\mathscr{R}_{\mathrm{L}}$ (indexed with greek indices) to those in $\mathscr{R}_{\mathrm{u}}$ (indexed with hatted latin indices), e.g.

$$
\hat{p}^{\hat{a}}=e^{\hat{a}}{ }_{\mu}(\mathrm{x}) p^{\mu}, \quad p^{\mu}=e_{\hat{a}}^{\mu}(\mathrm{x}) \hat{p}^{\hat{a}},
$$

with $e_{\hat{a}}^{\mu}(\mathrm{x})$ the inverse vierbein. See [5] for details. For non-Cartesian or curved geometries, an intermediate reference frame $\overline{\mathscr{R}}_{\mathrm{L}}$ must be defined, indexed by barred latin letters, and correspond- 
ing vierbein $e_{\mathrm{L}}{ }^{\bar{a}}$. This frame sets up a locally inertial frame at zero velocity at each spacetime point. If the background metric is written $g_{\mu \nu}(\mathrm{x})$, and the Minkowski metric $\eta_{\overline{a c}}$, then $e_{\mathrm{L}}{ }_{\mu}{ }_{\mu}$ can be obtained from:

$$
\eta_{\overline{a c}} e_{\mathrm{L}}^{\bar{a}}{ }_{\mu}(\mathrm{x}) e_{\mathrm{L}}^{\bar{c}}{ }_{v}(\mathrm{x})=g_{\mu v}(\mathrm{x}) .
$$

Then, because $\overline{\mathscr{R}}_{\mathrm{L}}$ and $\mathscr{R}_{\mathrm{u}}$ are locally inertial reference frames defined at the same spacetime point, they can be related one to the other through a Lorentz transform corresponding to a pure (de-)boost along the four-velocity $\bar{u}^{\bar{a}}$ (as defined in $\overline{\mathscr{R}}_{\mathrm{L}}$ ), with $\bar{u}^{\bar{a}}=e_{\mathrm{L}}{ }_{\mu}^{\bar{a}} u^{\mu}$. Writing this transform as $\Lambda^{\hat{a}}{ }_{\bar{c}}$, the vierbein $e^{\hat{a}} \mu(\mathrm{x})$ is eventually obtained as

$$
e^{\hat{a}}{ }_{\mu}(\mathrm{x})=\Lambda_{\bar{c}}^{\hat{a}}(\mathrm{x}) e_{\mathrm{L}}{ }^{\bar{c}} \mu(\mathrm{x}) .
$$

The Ricci rotation coefficients $\widehat{\Gamma}_{\hat{b} \hat{c}}^{\hat{a}}$ characterize the space-time dependence of the vierbein,

$$
\widehat{\Gamma}_{\hat{b} \hat{c}}^{\hat{a}}=-e^{\beta}{ }_{\hat{b}} e_{\hat{c}}^{\gamma} \nabla_{\gamma} e_{\beta}^{\hat{a}},
$$

and the symbol $\nabla_{\gamma}$ (written in short as $; \gamma$ in the following) represents a covariant derivative with respect to the metric $g_{\mu \nu}(\mathrm{x})$.

The connection $\widehat{\Gamma}_{\hat{b} \hat{c}}^{\hat{a}}$ enters the equation of motion in $\mathscr{R}_{\mathrm{u}}$ :

$$
\frac{\mathrm{d} \hat{p}^{\hat{a}}}{\mathrm{~d} \tau}=\frac{q}{m} \widehat{F}_{\hat{b}}^{\hat{a}} \hat{p}^{\hat{b}}-\widehat{\Gamma}_{\hat{b} \hat{c}}^{\hat{a}} \frac{\hat{p}^{\hat{b}} \hat{p}^{\hat{c}}}{m} .
$$

Here, $\widehat{F}^{\hat{a}}{ }_{\hat{b}}$ denotes the electromagnetic strength tensor in the (locally inertial) comoving frame and $\tau$ represents proper time. Because $\widehat{F}_{\hat{b}}=0$ by construction (vanishing electric field), the energy of the particle, $p^{\hat{0}}$, evolves only through the influence of the effective gravity, that derives from the non-inertial nature of the accelerated frame $\mathscr{R}_{\mathrm{u}}$. Equations (2.4) and (2.5) illustrate the fact that a spacetime dependence of the plasma four-velocity $\mathrm{u}$ is mandatory to achieve energization.

In the laboratory frame, the rate of energy gain of a particle can be obtained as

$$
\left\langle\frac{\Delta p^{t}}{\Delta t}\right\rangle=\lim _{\Delta t \rightarrow+\infty} \frac{1}{\Delta t}\left\langle e^{t}(\Delta \tau) \int_{0}^{\Delta \tau} \mathrm{d} \tau_{1} \frac{\mathrm{d} \hat{p}^{\hat{a}}}{\mathrm{~d} \tau_{1}}\right\rangle
$$

The explicit definition of the average $\langle\ldots\rangle$ depends on the case at hand, and must be specified with care, of course. Similarly, one can define a second-order moment to characterize diffusion processes,

$$
\left\langle\frac{\Delta p^{t} \Delta p^{t}}{\Delta t}\right\rangle=\lim _{\Delta t \rightarrow+\infty} \frac{1}{\Delta t}\left\langle e^{t} \hat{a}(\Delta \tau) e_{\hat{b}}^{t}(\Delta \tau) \int_{0}^{\Delta \tau} \mathrm{d} \tau_{1} \int_{0}^{\Delta \tau} \mathrm{d} \tau_{2} \frac{\mathrm{d} \hat{p}^{\hat{a}}}{\mathrm{~d} \tau_{1}} \frac{\mathrm{d} \hat{p}^{\hat{b}}}{\mathrm{~d} \tau_{2}}\right\rangle .
$$

Note that $\Delta t$ and $\Delta \tau$ should not be confused; they are related by

$$
\Delta t=\int_{0}^{\Delta \tau} \mathrm{d} \tau u^{t}
$$

The following provides three concrete applications to cases of interest, to illustrate the above method. 


\section{Applications}

Here, I consider in turn the acceleration of particles through scattering in a magnetized wind emitted by a compact object (Sec. 3.1), through scattering in the velocity field in the vicinity of a black hole (Sec. 3.1), and finally through their stochastic interactions with velocity structures in a magnetized turbulence (Sec. 3.3).

\subsection{Stochastic unipolar induction}

Consider a magnetized radial outflow characterized by its four-velocity $u^{\mu}(r, \theta)=\left\{u^{t}(r, \theta), u^{r}(r, \theta), 0,0\right\}$ in spherical coordinates, assumed to originate from some compact object. The magnetic field is transverse to the flow, but neither its magnitude nor its strength needs to be specified here. It suffices to assume that the particle has a finite mean free path $c t_{\mathrm{s}}$ in this structure ( $t_{\mathrm{s}}$ representing the scattering time), and for the sake of simplicity, this mean free path is assumed to be isotropic in the locally inertial frame. To compute Eqs. (2.6) and (2.7), one first needs to express the connection. Its non-vanishing components are:

$$
\widehat{\Gamma}_{\hat{1} \hat{0}}^{\hat{0}}=\frac{u^{r} u^{r}, r}{u^{t}}, \quad \widehat{\Gamma}_{\hat{1} \hat{1}}^{\hat{0}}=u_{, r}^{r}, \quad \widehat{\Gamma}_{\hat{1} \hat{2}}^{\hat{O}}=\frac{u^{r}, \theta}{r u^{t}}, \quad \widehat{\Gamma}_{\hat{2} \hat{2}}^{\hat{0}}=\frac{u^{r}}{r}, \quad \widehat{\Gamma}_{\hat{\mathrm{z}} \hat{\mathrm{z}}}^{\hat{0}}=\frac{u^{r}}{r} .
$$

In a rather straightforward way, one then derives

$$
\left\langle\frac{\mathrm{d} \hat{p}^{\hat{0}}}{\mathrm{~d} \tau}\right\rangle=-\frac{1}{3} \frac{\hat{p}^{2}}{m} \nabla \cdot \boldsymbol{u} .
$$

Here, the meaning of the averages is to assume that the direction of the particle momentum in the locally inertial frame is random. It can thus be seen as an average over the particle distribution function over time scales much larger than the particle mean free path. It reproduces the correct law of evolution for a fluid (corresponding to zero mean free path particles), describing cooling (resp. heating) through expansion (resp. contraction).

Particles with a non-zero mean free path can explore the flow in different directions, and therefore interact with electric fields of different directions and magnitudes, because of the nonuniformity of $u(x)$. Such particles can therefore gain energy in a stochastic way, as characterized by the second order moment

$$
\Delta^{\hat{0} \hat{0}}=\int \mathrm{d} \tau_{1} \mathrm{~d} \tau_{2}\left\{\left\langle\frac{\mathrm{d} \hat{p}^{\hat{0}}}{\mathrm{~d} \tau_{1}} \frac{\mathrm{d} \hat{p}^{\hat{0}}}{\mathrm{~d} \tau_{2}}\right\rangle-\left\langle\frac{\mathrm{d} \hat{p}^{\hat{0}}}{\mathrm{~d} \tau_{1}}\right\rangle\left\langle\frac{\mathrm{d} \hat{p}^{\hat{0}}}{\mathrm{~d} \tau_{2}}\right\rangle\right\},
$$

which reads here

$$
\Delta^{\hat{0} \hat{0}}=2 \widehat{\Delta t} \frac{\hat{p}^{2} \hat{t}_{\mathrm{s}}}{18 r^{2}}\left[4 u^{r 2}+2\left(r u_{, r}^{r}\right)^{2}+\frac{u^{r}, \theta^{2}}{u^{t^{2}}}+6 \frac{\left(r u^{r}, r\right)^{2}}{u^{t^{2}}}\right] .
$$

As expected, this term vanishes for particles of zero mean free path. Given the flow geometry $u^{\mu}(\mathrm{x})$, and $\hat{t}_{\mathrm{s}}$, one can solve the above equations to obtain the evolution of the particle distribution function in a Fokker-Planck manner.

The terms that appear in these first and second-order moments in Eqs. (3.2) and (3.4) can in principle be obtained from the transport equation for the particle distribution, which is itself 
derived in a perturbative manner from the Vlasov equation, at the price, however, or intensive algebra, see [6, 7]. The regular, also termed adiabatic, term in Eq. (3.2), then emerges in front of $\partial f / \partial \hat{p}$, while the second-order terms of Eq. (3.4) appear in front of $\partial^{2} f / \partial \hat{p}^{2}$, and correspondingly characterize the stochastic energization terms. Such terms involve the shear tensor $\sigma_{\alpha \beta}=u_{(\alpha ; \beta)}+u_{(\alpha} u^{\mu} u_{\beta) ; \mu}-\frac{1}{3} u_{; \mu}^{\mu} h_{\alpha \beta}$, with the short-hand notation $u_{(i, j)}=\left(u_{i, j}+u_{j, i}\right) / 2$ and $h_{\alpha \beta}=g_{\alpha \beta}+u_{\alpha} u_{\beta}$ the metric of hypersurfaces orthogonal to $u^{\mu}$. They also involve the acceleration term $a_{\alpha \beta}=-u_{\beta} u^{\mu} u_{\alpha ; \mu}$.

\subsection{Acceleration in black hole environments}

As a second example, consider a particle scattering in a magnetized turbulence in the vicinity of a black hole. Doing so, the particle explores both the sheared velocity flow, but also the gravitational potential and both contribute to its energization. In principle, the particle can also gain energy through its interaction with the turbulence, if the latter contains fast moving scattering centers, in the standard Fermi-II process. For the sake of simplicity, this source of scattering is ignored here; it will, however, be considered in Sec. 4. Note that, as the velocity of the scattering centers, e.g. the Alfvén velocity $\beta_{\mathrm{A}}$, drops to zero, the turbulence ceases to provide acceleration, but it still contributes to scattering and transport.

For simplicity, I consider here the case of a circular orbit around a Schwarzschild black hole. The metric is $g_{\mu \nu}=\operatorname{diag}\left\{-\left[1-r_{\mathrm{H}} / r\right],\left[1-r_{\mathrm{H}} / r\right]^{-1}, r^{2}, r^{2} \sin ^{2} \theta\right\}$, where $r_{\mathrm{H}}=2 G M / c^{2}$ represents the horizon radius in terms of the black hole mass $M$. The circular orbit is described by $u^{\mu}=\gamma_{u}(r)\{1,0,0, \Omega(r)\}$. Here, the laboratory frame is that in which the black hole lies at rest. The angular momentum of the flow is $\ell_{u}=u_{\phi} / u_{t}=r^{2} \sin ^{2} \theta \Omega /\left(1-r_{\mathrm{H}} / r\right)$.

To simplify the expressions that follow, consider the orbit on the equator. Then, the non-zero time components of the connection are:

$$
\widehat{\Gamma}_{\hat{1} \hat{0}}^{\hat{0}}=\frac{r^{3} r_{\mathrm{H}}-2 \ell_{u}^{2}\left(r-r_{\mathrm{H}}\right)^{2}}{2 r^{3 / 2} \sqrt{r-r_{\mathrm{H}}}\left[r^{3}-\ell_{u}^{2}\left(r-r_{\mathrm{H}}\right)\right]}, \widehat{\Gamma}_{\hat{1} \hat{\jmath}}^{\hat{0}}=-\frac{\ell_{u}\left(2 r-3 r_{\mathrm{H}}\right)}{2\left[r^{3}-\ell_{u}^{2}\left(r-r_{\mathrm{H}}\right)\right]}, \widehat{\Gamma}_{\hat{3} \hat{1}}^{\hat{0}}=-\frac{\ell_{u}\left(2 r-3 r_{\mathrm{H}}\right)}{2\left[r^{3}-\ell_{u}^{2}\left(r-r_{\mathrm{H}}\right)\right]} .
$$

From this, one derives

$$
\left\langle\frac{\mathrm{d} \hat{p}^{\hat{0}}}{\mathrm{~d} \tau}\right\rangle=0
$$

indicating that on average, the particle does not gain or lose energy along its circular orbit, as expected. Here as well, the average is taken over the random orientation of the particle momentum in the comoving frame. However, the second-order moment does not vanish, and it can be written in the compact way:

$$
\Delta^{\hat{0} \hat{0}}=2 \widehat{\Delta t} \frac{\hat{p}^{2} \hat{t}_{\mathrm{s}}}{3 r^{2}\left[1+\left(1-r_{\mathrm{H}} / r\right) \ell_{u}^{2} / r^{2}\right]^{2}}\left\{\left[\left(1-\frac{r_{\mathrm{H}}}{r}\right)^{3 / 2} \frac{\ell_{u}^{2}}{r^{2}}-\left(1-\frac{r_{\mathrm{H}}}{r}\right)^{-1 / 2} \frac{r_{\mathrm{H}}}{r}\right]^{2}+\frac{\sqrt{2}}{6} r^{4} \Omega_{, r}^{2}\right\} .
$$

This term involves three contribution: the first two, whose difference is squared, respectively correspond to the centrifugal term $\propto \ell_{u}^{2}$ and the gravitational potential, adding up to form the total force potential acting on the particle, while the third describes the shear contribution. The above expression reduces to that obtained in the flat spacetime limit $\left(r_{\mathrm{H}} \rightarrow 0\right)$ in Ref. [8], up to a numerical prefactor (the difference is tied to how one defines $\hat{t}_{\mathrm{s}}$ ). 
To the author's knowledge, the above expression has not been published before and it allows to compute the heating/acceleration rate of an ensemble of particles orbiting a black hole on tight orbits. Deriving it from a full general relativistic transport equation is a priori possible, albeit at the price of cumbersome algebra. Finally, it should be noted that, as Eq. (3.4) previously, the present derivation assumes $\hat{t}_{\mathrm{s}} \ll r$, meaning that the particle scatters around its circular orbit, but remains tied to it on average.

\subsection{Stochastic acceleration in a magnetized turbulence}

As a last application, consider the acceleration of particles in a magnetized turbulent bath. In the traditional approach, the turbulence is described as a sum of plane waves and one relies on quasilinear theory, adding up the random force contributions that the particle experiences along its $u$ nperturbed trajectory along the large-scale magnetic field. Energy gain then results from waveparticle gyro-resonant (or Landau-resonant) interactions [2]. However, whether one can actually describe a turbulence as a bath of waves or not, and whether such resonances survive in modern turbulence theories $[9,10]$, remains a subject of debate.

Meanwhile, particles can gain energy through nonresonant processes in a generic turbulence. Consider for instance the term $\boldsymbol{\nabla} \cdot \mathbf{u}$ that enters Eq. (3.2): there it provides net cooling because it has a definite sign, while in a turbulent bath, a particle can encounter regions of either sign. One may thus expect a null contribution on average, but a non-zero variance leading to diffusion in momentum space. How the velocity pattern affects the particle momentum is entirely encoded in Eq. (2.5) above.

Such nonresonant diffusion processes have been discussed in the subrelativistic limit, starting with Refs. [11, 12], but not in the relativistic limit where they may play an important role in promoting fast acceleration. The formalism is ideally suited to characterize such processes and their influence. To simplify the discussion, one makes a number of simplifying assumptions:

1. The magnitude of the flow four-velocity is assumed uniform in time and space, i.e. $u_{, \alpha}^{t}=0$, but its direction in space is assumed to form a Gaussian random process, characterized by the correlation time $\Omega^{-1}$ and length scale $K^{-1}$.

2. The direction of the particle momentum also follows a random process, characterized by its correlation time $t_{\mathrm{s}}$.

3. Isotropicity is implicitly assumed in the local comoving frame.

Each of these assumptions could be relaxed, of course.

The following omits the details of the calculations, which can be found in [5], but it provides the main steps. One first decomposes the velocity field into its 3D expansion $(\theta)$, acceleration $(\boldsymbol{a})$, $\operatorname{shear}\left(\sigma_{i j}\right)$ and vorticity $\left(\omega_{i j}\right)$ components: $\theta=\nabla \cdot \mathbf{u}, \boldsymbol{a}=\boldsymbol{u}_{, t}, \sigma_{i j}=u_{(i, j)}-\frac{1}{3} \eta_{i j} \theta$ and $\omega_{i j}=u_{[i, j]}$. Assuming that these quantities are not correlated one to another, correlation functions involving two derivatives of the velocity field can be decomposed into the sum of the correlation functions of each of these quantities, e.g.

$$
\left\langle u^{i, j}\left(\mathrm{x}_{1}\right) u_{i, j}\left(\mathrm{x}_{2}\right)\right\rangle=\frac{1}{3}\left\langle\theta^{2}\right\rangle \mathscr{C}_{\theta}\left(\mathrm{x}_{1} ; \mathrm{x}_{2}\right)+\left\langle\sigma^{2}\right\rangle \mathscr{C}_{\sigma}\left(\mathrm{x}_{1} ; \mathrm{x}_{2}\right)+\left\langle\omega^{2}\right\rangle \mathscr{C}_{\omega}\left(\mathrm{x}_{1} ; \mathrm{x}_{2}\right)
$$


where $\mathscr{C}_{\theta}\left(\mathrm{x}_{1} ; \mathrm{x}_{2}\right)$ represents the correlation function of $\theta$ (normalized to integral unity), and similarly for the other terms. The above decomposition involves the fluctuation amplitudes $\left\langle\sigma^{2}\right\rangle=$ $\left\langle\sigma^{i j} \sigma_{i j}\right\rangle$ and similarly for the other terms.

The calculation scheme is then the following:

1. one first expresses the connection in terms of the velocity field and its derivatives, then plugs in Eq. (2.5) into and (2.6) and (2.7). The first order moment vanishes exactly, since $\langle\theta\rangle=0$.

2. The expression for the second-order moment can be simplified, using Wick's theorem to break down the correlators of the random fields down to two-point functions.

In this way, one obtains to lowest order in $u^{2}$, in the sub-relativistic limit, a simple, closed formula:

$$
\begin{aligned}
\left\langle\Delta p^{2}\right\rangle=p^{2} \int \mathrm{d} t_{1} \mathrm{~d} t_{2} & \left\{\left\langle\theta^{2}\right\rangle \frac{2}{3} \mathscr{C}_{\theta}\left(t_{1}, t_{2}\right) \mathscr{C}_{p}\left(t_{1}, t_{2}\right)^{2}+\left\langle a^{2}\right\rangle \mathscr{C}_{a}\left(t_{1}, t_{2}\right) \mathscr{C}_{p}\left(t_{1}, t_{2}\right)\right. \\
+ & \left.\frac{2}{5}\left\langle\sigma^{2}\right\rangle \mathscr{C}_{\sigma}\left(t_{1}, t_{2}\right) \mathscr{C}_{p}\left(t_{1}, t_{2}\right)^{2}\right\} .
\end{aligned}
$$

To simplify it further, consider the limit $\Omega \rightarrow 0$ (corresponding to perturbations that never decorrelate in time), and assume first the limit $t_{\mathrm{S}} \ll K^{-1}$. The explicit integration of the above equation then provides, to lowest order:

$$
\frac{\left\langle\Delta p^{2}\right\rangle}{2 \Delta t}=\frac{\sqrt{2}}{3} p^{2} t_{\mathrm{s}}\left(\left\langle\theta^{2}\right\rangle+\frac{3}{5}\left\langle\sigma^{2}\right\rangle+\frac{3}{\sqrt{2}}\left\langle a^{2}\right\rangle\right)
$$

which shows that the compressive, shear and acceleration components of the turbulence contribute in about equal amounts to momentum diffusion. Given that $\left\langle\theta^{2}\right\rangle \sim K^{2}\left\langle u^{2}\right\rangle_{\text {compr. }}$, where $\left\langle u^{2}\right\rangle_{\text {compr. }}$ represents the four-velocity fluctuation power in compressible modes, and similarly for the other quantities, Eq. (3.10) above suggests that the acceleration timescale takes the form:

$$
t_{\mathrm{acc}}=\frac{p^{2}}{\left\langle\Delta p^{2} / 2 \Delta t\right\rangle} \sim \frac{1}{\left\langle u^{2}\right\rangle} \frac{L^{2}}{t_{\mathrm{s}}}
$$

with $L=K^{-1}$ the typical scale of the velocity flow, $\left\langle u^{2}\right\rangle$ symbolizing the fluctuation amplitude associated to the combination of compressive, shear and acceleration motions indicated in Eq. (3.10). Note also that the present calculation assumes a spectrum strongly peaked on a single scale; this will be generalized to a more realistic spectrum further below. This acceleration timescale can be interpreted as follows: it takes a time $L^{2} / t_{\mathrm{s}}$ for the particle to explore the coherence length of the turbulence, while traveling in a diffusive manner (recall $t_{\mathrm{s}} \ll L$ here), and thereby to experience a decorrelated force that promotes diffusion in momentum space. For reference, in the Fermi model, a particle interacting with structures moving at velocity $\left\langle u^{2}\right\rangle^{1 / 2}$ with interaction time $t_{\text {int }}$ gets accelerated on a timescale $t_{\text {acc }} \sim t_{\text {int }} /\left\langle u^{2}\right\rangle$. Here, therefore, $t_{\text {int }} \sim L^{2} / t_{\mathrm{s}}$, in sharp contrast with the predictions from quasilinear theory for resonant wave-particle interactions, which give $t_{\text {int }} \sim t_{\mathrm{s}}$.

In the opposite limit $t_{\mathrm{s}} \gg K^{-1}$, one derives:

$$
\frac{\left\langle\Delta p^{2}\right\rangle}{2 \Delta t}=\frac{4}{\pi} p^{2} \frac{1}{K^{2} t_{\mathrm{s}}}\left(\left\langle\theta^{2}\right\rangle+\frac{3}{5}\left\langle\sigma^{2}\right\rangle+\frac{3}{2}\left\langle a^{2}\right\rangle\right)
$$


indicating that the acceleration timescale now reads $t_{\mathrm{acc}} \sim t_{\mathrm{s}} /\left\langle u^{2}\right\rangle$. One thus recovers in this regime $t_{\text {int }} \sim t_{\mathrm{s}}$, because the particle has already crossed many coherent lengths of the turbulence in a time interval $t_{\mathrm{s}}$.

The above can be generalized to the ultra-relativistic regime. In this case, the evolution of the momentum takes the following form:

$$
\left\langle\Delta p^{2}\right\rangle \sim\langle p\rangle^{2} \sim \gamma_{u}^{4} p_{0}^{2} e^{2 \Delta t / t_{p p}},
$$

with $p_{0}=p^{t}(0)$. The term $\gamma_{u}^{4}$ results from a first-order Fermi process that is associated with the bulk motion of the turbulence in the laboratory frame. The term $t_{p p}$ represents the timescale for acceleration through stochastic interactions with the turbulence. The expressions for $t_{p p}$ in the limits $t_{\mathrm{S}} \ll K^{-1}$ and $t_{\mathrm{s}} \gg K^{-1}$ take forms similar to those above for $t_{\mathrm{acc}}$, except that the vorticity now contributes as well. Explicitly:

$$
\begin{array}{lc}
t_{p p}=\frac{t_{\mathrm{s}}^{-1}}{0.87\left\langle\theta^{2}\right\rangle+0.40\left\langle\sigma^{2}\right\rangle+0.52\left\langle\omega^{2}\right\rangle+2.4\left\langle a^{2}\right\rangle} & \left(t_{\mathrm{s}} \ll K^{-1}\right) \\
t_{p p}=\frac{K^{2} t_{\mathrm{s}}}{1.9\left\langle\theta^{2}\right\rangle+0.94\left\langle\sigma^{2}\right\rangle+0.90\left\langle\omega^{2}\right\rangle+7.3\left\langle a^{2}\right\rangle} & \left(t_{\mathrm{s}} \gg K^{-1}\right) .
\end{array}
$$

The above expressions have been derived under the assumption that the power spectrum of the velocity fluctuations is sharply peaked around a single scale $L=K^{-1}$. They may, however, be generalized to the case of a broadband spectrum, as has been done in Ref. [13]. The idea is to split the cascade into two regions, the small-scale part with $k \gg t_{\mathrm{s}}$, and the large-scale part with $k \ll t_{\mathrm{s}}$, then to use the corresponding expressions derived above, for each. First, one decomposes the four-velocity fluctuations as

$$
\left\langle\delta u^{2}\right\rangle=\int_{\ln k_{\min }} \mathrm{d} \ln k\left\langle\left|\delta u_{k}\right|^{2}\right\rangle_{k},
$$

with $\left\langle\left|\delta u_{k}\right|^{2}\right\rangle_{k} \propto k^{1-q_{u}}$, where $q_{u}$ represents the 1D power spectrum index of the velocity field, and $k_{\min }$ the smallest wavenumber of the cascade (i.e., the inverse of the outer scale). Then, the various contributions of compressive modes, acceleration, shear and vorticity on a scale $k$ are written, e.g. for the compressive part

$$
\left\langle\theta^{2}\right\rangle_{k}=\alpha_{\theta} k^{2}\left\langle\left|\delta u_{k}\right|^{2}\right\rangle_{k}
$$

For ease of notation and simplification, the various contributions of the compressive modes, shear etc. are summed up in one global term written $\alpha k^{2}\left\langle\left|\delta u_{k}\right|^{2}\right\rangle_{k}$, although it should be kept in mind that the exact combination depends on the properties of the turbulence. The notation that follows is thus schematic. Interestingly, both contributions from the large scale part of the cascade, above $t_{\mathrm{S}}$, and from the small scale part, give similar contributions to $\left\langle\Delta p^{2} / 2 \Delta t\right\rangle$, and one ends up with

$$
\frac{\left\langle\Delta p^{2}\right\rangle}{2 \Delta t} \sim p^{2}\left(t_{\mathrm{s}} k_{\min }\right)^{q_{u}-2}\left\langle\delta u^{2}\right\rangle \alpha k_{\min } .
$$

The above relationship between the diffusion coefficient and the scattering timescale departs markedly from the quasilinear prediction $D_{p p} \propto 1 / t_{\mathrm{s}}$. Furthermore, because $\left|q_{u}-2\right|$ generically is a small quantity, and because $t_{\mathrm{s}}$ generally is a mild (increasing) function of the momentum, the above suggests that $D_{p p} \sim p^{2} k_{\min }\left\langle\delta u^{2}\right\rangle$, in a reasonable approximation. Interestingly, this scaling has been observed in recent particle-in-cell simulations of relativistic turbulence [14, 15]. 


\section{Conclusions}

This paper, given at the occasion of the HEPRO VII (High Energy Processes in Relativistic Outflows) meeting (Barcelona, 2019), and based on [5], introduces a new formalism to describe the acceleration of particles in highly conducting astrophysical plasmas. This description relies on the observation that the electric field exactly vanishes in the instantaneous frame of rest of the plasma, as a virtue of ideal Ohm's law (infinite conductivity), so that, in that frame, the Lorentz force only contributes to pitch angle scattering, not energization. If the velocity field is not uniform throughout space, this frame is not inertial, hence the particle is subject to an effective gravity that provides energy gains or losses. To characterize the physics of acceleration, one follows the journey of the particle in phase space in mixed frames, tracking the particle position in a laboratory frame and the momentum in the sequence of locally inertial frames (instantaneous frames of rest) that the particle visits. This generalizes to an arbitrary flow pattern the well-known scheme of calculating the energy gain through Lorentz boosts to and from the rest frame of a scattering center, which can be used only in specific configurations, as diffusive shock acceleration for instance.

The present formalism, because it makes use of general covariance, can be applied to all flow configurations in any geometry, i.e. sub- to ultra-relativistic, Cartesian or non-Cartesian geometries, flat or curved spacetime. Three concrete examples have been presented here as applications: stochastic acceleration in an expanding magnetized radial wind, gravito-centrifugo-shear acceleration including strong-field effects in the vicinity of a black hole and, finally, non-resonant acceleration in a magnetized turbulence. Each represents an original calculation, because such evolved configurations cannot be easily addressed with other formalisms for Fermi acceleration.

Acknowledgements: This work has been financially supported by the ANR-14-CE33-0019 $\mathrm{MACH}$ project.

\section{References}

[1] E. Fermi, On the Origin of the Cosmic Radiation, Phys. Rev. 75 (1949) 1169.

[2] C. F. Kennel and F. Engelmann, Velocity Space Diffusion from Weak Plasma Turbulence in a Magnetic Field, Phys. Fl. 9 (1966) 2377.

[3] L. O. Drury, An introduction to the theory of diffusive shock acceleration of energetic particles in tenuous plasmas, Rep. Prog. Phys. 46 (1983) 973.

[4] F. M. Rieger, An Introduction to Particle Acceleration in Shearing Flows, Galaxies 7 (2019) 78 [1909.07237].

[5] M. Lemoine, Generalized Fermi acceleration, Phys. Rev. D 99 (2019) 083006 [1903. 05917 ].

[6] G. M. Webb, Relativistic transport theory for cosmic rays, Astrophys. J. 296 (1985) 319.

[7] G. M. Webb, The diffusion approximation and transport theory for cosmic rays in relativistic flows, Astrophys. J. 340 (1989) 1112.

[8] F. M. Rieger and K. Mannheim, Particle acceleration in rotating and shearing jets from AGN, Astron. Astrophys. 396 (2002) 833 [astro-ph/ 0210286 ]. 
[9] B. D. G. Chandran, Scattering of Energetic Particles by Anisotropic Magnetohydrodynamic Turbulence with a Goldreich-Sridhar Power Spectrum, Phys. Rev. Lett. 85 (2000) 4656 [astro-ph/0008498].

[10] H. Yan and A. Lazarian, Scattering of Cosmic Rays by Magnetohydrodynamic Interstellar Turbulence, Phys. Rev. Lett. 89 (2002) B1102 [arXiv:astro-ph/0205285].

[11] A. M. Bykov and I. N. Toptygin, Cosmic Rays Acceleration by Large Scale Compressible and Incompressible Motions of Plasma, Int. Cosmic Ray Conf. 9 (1983) 313.

[12] V. S. Ptuskin, Cosmic-Ray Acceleration by Long-Wave Turbulence, Sov. Astron. Lett. 14 (1988) 255.

[13] C. Demidem, M. Lemoine and F. Casse, Particle acceleration in relativistic turbulence: a theoretical appraisal, arXiv e-prints (2019) arXiv:1909.12885 [1909.12885].

[14] K. Wong, V. Zhdankin, D. A. Uzdensky, G. R. Werner and M. C. Begelman, First-principles demonstration of diffusive particle acceleration in kinetic simulations of relativistic plasma turbulence, arXiv e-prints (2019) arXiv:1901.03439 [1901.03439].

[15] L. Comisso and L. Sironi, The interplay of magnetically-dominated turbulence and magnetic reconnection in producing nonthermal particles, arXiv e-prints (2019) arXiv:1909.01420 [1909.01420]. 